\title{
Localization properties of a tight-binding electronic model on the Apollonian network
}

\author{
A. L. Cardoso, ${ }^{1}$ R. F. S. Andrade, ${ }^{1,2}$ and A. M. C. Souza ${ }^{3}$ \\ ${ }^{1}$ Instituto de Física, Universidade Federal da Bahia, 40210-210, Salvador, Brazil \\ ${ }^{2}$ Computational Physics, IfB, ETH-Hönggerberg, Schafmattstr. 6, 8093, Zürich, Switzerland \\ ${ }^{3}$ Departamento de Física, Universidade Federal de Sergipe, 49.100-000, São Cristovão, Brazil
}

(Received 23 September 2008; published 4 December 2008)

\begin{abstract}
An investigation on the properties of electronic states of a tight-binding Hamiltonian on the Apollonian network is presented. This structure, which is defined based on the Apollonian packing problem, has been explored both as a complex network and as a substrate on the top of which physical models can be defined. The Schrödinger equation of the model, which includes only nearest-neighbor interactions, is written in a matrix formulation. In the uniform case, the resulting Hamiltonian is proportional to the adjacency matrix of the Apollonian network. The characterization of the electronic eigenstates is based on the properties of the spectrum, which is characterized by a very large degeneracy. The $2 \pi / 3$ rotation symmetry of the network and large number of equivalent sites are reflected in all eigenstates, which are classified according to their parity. Extended and localized states are identified by evaluating the participation rate. Results for other two nonuniform models on the Apollonian network are also presented. In one case, interaction is considered to be dependent of the node degree, while in the other one random on-site energies are considered.
\end{abstract}

DOI: 10.1103/PhysRevB.78.214202

PACS number(s): 71.23.An, 73.20.At, 73.20.Fz, 89.75.Hc

\section{INTRODUCTION}

Modeling physical systems on substrates without translation invariance provides useful insights for the understanding of disordered systems. ${ }^{1}$ Quite recently, the rapid development of complex network theory ${ }^{2}$ has motivated the investigation of physical models on such substrates for the purpose of identifying new aspects that may stem from geometrical constructs. ${ }^{3,4}$ Indeed, the characterization of their physical properties has shown that new thermodynamic, magnetic, and transport features do emerge. In several situations, ${ }^{5}$ they can become quite distinct from those obtained by other disordered models.

The Apollonian network (AN) (Ref. 6) appears in this scenario as an interesting structure in the sense that it shares several properties of complex networks, but on the other hand it is defined by strict geometrical rules. This particular feature makes it possible to explore scale-invariance properties together with numerical methods in the investigation of distinct models. Its specific geometric features induce the emergence of nontypical behavior in thermodynamic and magnetic properties of spin systems, ${ }^{7,8}$ avalanche distribution in sand pile models, ${ }^{9}$ and so on.

The recent creation of a synthetic nanometer-scale Sierpinski hexagonal gasket, a self-similar fractal macromolecule, ${ }^{10}$ has highly motivated the area of scaleinvariant networks. This perspective raises the question about electronic systems on these networks.

The purpose of this work is to investigate tight-binding electronic models on the AN, focusing on the localized vs extended nature of their wave functions. This characterization follows a previous analysis of the properties of the eigenvalue spectrum of the adjacency matrix (AM) of the AN. ${ }^{11}$ The AM spectrum is a very important network signature and, as such, its properties have been detailed explored within graph theory. In particular, great attention has been devoted to understanding isospectral graphs that are not isomorphic. $^{12,13}$
As we will show, the Hamiltonian of the uniform tightbinding model can be written in terms of the AM, so that the energy eigenvalues are proportional to those of the AM. The previous identification of the structure of the spectrum detailed the presence of several distinct classes and highly degenerated subsets. The characterization of the localization properties of the wave function, based on the evaluation of the participation rate of each state, follows the classification scheme of different sets in the AM spectrum.

The paper is organized as follows. In Sec. II, we discuss the main features of the AN. We also introduce the tightbinding model and its matrix. We also indicate how the participation rate has been used to quantify the localization properties of the eigenstates. Section III brings a brief review of the most important properties of the uniform eigenvalue spectrum. Results for the three different models are presented in Sec. IV. Finally, Sec. V closes the paper with a summary of our main important results.

\section{TIGHT-BINDING MODELS ON AN}

ANs (Refs. 6 and 14) follow from the old problem of optimally filling a compact domain in the Euclidean plane by circles. ${ }^{15}$ The solution to this problem requires the successive addition of maximal tangent circle into the empty regions bounded by three previously placed circles. At a given generation $n$, the network $\mathrm{AN}_{n}$ is constructed by establishing a connection between the centers of all pairs of tangent circles. The solution to the packing problem requires the determination of the center and radius of each placed circle. For the AN, however, these details are not required, and we just need to assign the pairs of nodes that are connected. In this work, we consider the simplest situation, which arises by considering, at the zeroth generation $n=0$, three tangent circles with the same radius. The centers of which occupy the corners of a equilateral triangle (see Fig. 1). The number of sites $N(n)$ on the network increases according to $N(n)=\left(3^{n}+5\right) / 2$, 


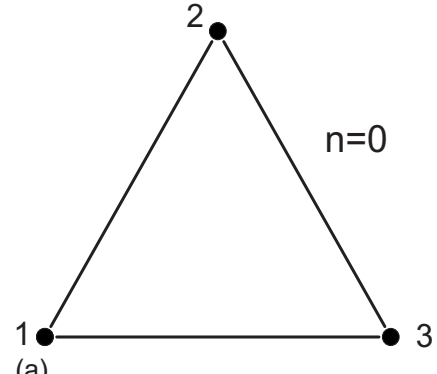

(a)

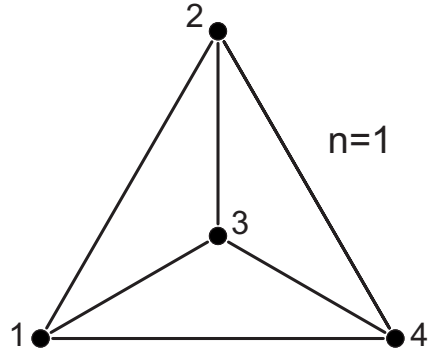

(b)

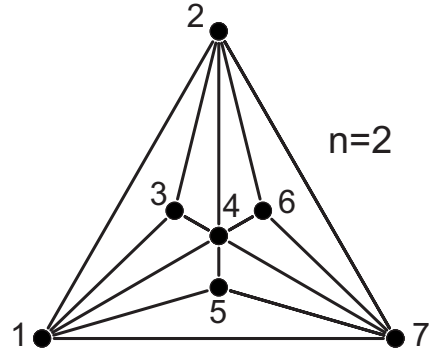

(c)

FIG. 1. Generations $n=0,1$, and 2 of AN. The adopted site numbering is the one introduced in Ref. 7.

while the number of edges $B(n)$ depends on $n$ as $B(n)$ $=\left(3^{n+1}+3\right) / 2$. In the limit $n \rightarrow \infty, B(n) / N(n) \rightarrow 3$, so that each node has, in the average, six neighbors.

The resulting AN shares several properties with other classes of complex network, including being scale free [the distribution $P(k)$ of node degree $k$ is a power law], small world (the mean minimal path $\langle\ell\rangle \sim \log N$ ), and hierarchical [the clustering coefficient of individual nodes $c(k)$ has a power law dependence on $k$ ]. Nevertheless, it has own features ${ }^{16}$ that preclude being put into the set of networks generated by known algorithms such as those proposed by Watts and Strogatz ${ }^{17}$ and Barabasi and Albert. ${ }^{18}$

The model considers that an electron is primarily bound to a network node $i$ with on-site energy $\epsilon_{i}$, but it is also allowed to hop from $i$ to a neighboring site $j$ one with a probability amplitude $V_{i, j}=V_{j, i}$, resulting from the overlap integral involving the two wave functions and interaction potential. For a given $n$, the corresponding Hamiltonian is written as

$$
H_{n}=\sum_{i=1}^{N(n)}|i\rangle \epsilon_{i}\left\langle i\left|+\sum_{(i, j)}\right| i\right\rangle V_{i, j}\langle j|,
$$

where $|i\rangle$ represents a Wannier function for an electron at position $i$ and $(i, j)$ denotes pairs of sites that are first neighbors.

The Hamiltonian (1) can be written in a matrix form, where the diagonal elements $H_{i, i}=\epsilon_{i}$, while off-diagonal elements $H_{i, j}$ are set to $V_{i, j}$ or 0 , depending on whether sites $i$ and $j$ are neighbors or not. The AN sites are numbered according to a previously introduced scheme, which is reproduced in Fig. 1 for $n=0,1$, and 2. When we go from generation $n$ to $n+1$, the whole $n$th lattice is first squeezed into the region limited by two outer vertices and the site at the geometrical center of the triangle. After this first step, two identical copies of the squeezed lattice, rotated by $2 \pi / 3$ and $4 \pi / 3$, are added to the regions that have been emptied. In this numbering, two of the outer vertices always take the numbers 1 and 2, while the third one assumes the number $N(n)$ for generation $n$. As a consequence, the central node receives always the number $N(n-1)$. The matrix representation of $\mathrm{H}_{2}$ is

$$
H_{2}=\left(\begin{array}{ccccccc}
\epsilon_{1} & V_{1,2} & V_{1,3} & V_{1,4} & V_{1,5} & 0 & V_{1,7} \\
V_{2,1} & \epsilon_{2} & V_{2,3} & V_{2,4} & 0 & V_{2,6} & V_{2,7} \\
V_{3,1} & V_{3,2} & \epsilon_{3} & V_{3,4} & 0 & 0 & 0 \\
V_{4,1} & V_{4,2} & V_{4,3} & \epsilon_{4} & V_{4,5} & V_{4,6} & V_{4,7} \\
V_{5,1} & 0 & 0 & V_{5,4} & \epsilon_{5} & 0 & V_{5,7} \\
0 & V_{6,2} & 0 & V_{6,4} & 0 & \epsilon_{6} & V_{6,7} \\
V_{7,1} & V_{7,2} & 0 & V_{7,4} & V_{7,5} & V_{7,6} & \epsilon_{7}
\end{array}\right) .
$$

The simple uniform model corresponds to setting $\epsilon_{i}=\epsilon_{0}, \forall i$ and $V_{i, j}=V_{0}, \forall(i, j)$. If we let $E^{\prime}$ to be the eigenvalues of $H$, we can define normalized energies $E=\left(E^{\prime}-\epsilon_{0}\right) / V_{0}$, which can also be directly obtained by rewriting Eq. (2) and setting $\epsilon_{0}=0$ and $V_{0}=1$. In terms of these variables, matrix (2) coincides exactly with the AM of the AN. Thus, the eigenenergies of the homogeneous tight-binding model can be directly obtained from the AM spectrum discussed previously. For the sake of a simple notation, we will always refer, in the forthcoming discussion, to the redefined values of $E$. With the exception of the random on-site energy model, we restrict ourselves to investigate the situation in which $\epsilon_{0}=0$.

Nonuniform models can be defined by choosing other values either for the self-energies or the hopping integrals. In this work we consider a family of models defined by $\epsilon_{i}$ $=0, \forall i$ and $V(i, j)=1 / z^{\beta}$. Here, $z=\left(k_{i}+k_{j}\right) / 2$ is the average value of the node degrees $k$, and the value of $\beta$ selects a particular element of the set. The uniform model corresponds to setting $\beta=0$. Finally, the third model that aims to investigate the existence of Anderson transition ${ }^{19}$ in $\mathrm{AN}$ is defined by setting $V(i, j)=1$ for neighboring sites, while $\epsilon_{i}$ are chosen according to a probability distribution function.

The solution to the Schrödinger equation $H\left|\Psi_{E}\right\rangle=E\left|\Psi_{E}\right\rangle$ can be written in the basis of Wannier states, i.e., $\left|\Psi_{E}\right\rangle$ $=\sum_{i=1}^{N} a_{E, i}|i\rangle$. The site coefficients $a_{E, i}$ are the components of the normalized eigenvector corresponding to the eigenvalue $E$, which are obtained from the numerical diagonalization of the Hamiltonian matrices. The knowledge of the vector components $a_{i}$ and the possibility of evaluating it as a function of the subsequent generations $n$ suggest the use of the participation ratio $\xi$ to characterize the degree of localization of the electronic states. For normalized states, this often used measure is defined by 


$$
\xi_{E, n}=\frac{1}{\sum_{i=1}^{N(n)}\left|a_{E, i}\right|^{4}} .
$$

It can assume values in the range $[1, N]$, which correspond, respectively, to the extreme situations of completely localized $\left(a_{E, i}=1\right.$ for one single value of $i$ ) and extended states $\left(a_{E, i}=1 / \sqrt{N}, \forall i\right)$. Thus, it provides both an estimate for the localization length $\lambda$, as indications for extended states, for which $\xi$ should scale with $N(n)$. Since the model is defined in the limit $n \rightarrow \infty$, the localized or extended character of the Apollonian states of the infinite system can be inferred from the behavior of $\xi_{E, n}$ as a function of $n$.

Let us recall that the eigenstates of $H_{n}$ corresponding to a degenerate eigenvalue $\bar{E}$ are not univocally determined, as any linear combination of them is still a solution of the secular equation. Thus, it is inevitable that different algorithms used to evaluate the eigenvectors lead to different values of $\xi_{E, n}$. By performing numerical evaluation of all eigenvectors to a degenerated eigenvalue with two distinct routines, we find two different sets of eigenvectors and values of $\xi_{E, n}$. However, the localization properties remain the same.

\section{SPECTRUM PROPERTIES}

The evaluation of the eigenvalues must be performed with the help of numerical algorithms. The spectrum of the uniform model has been characterized in a detailed way. In this particular case, the presence of many eigenvalues with high degree of degeneracy, and the recurrent presence of the same eigenvalues in the successive increasing values of $n$, makes it possible to present a classification scheme for the eigenvalues $E$. According to it, for any value of $n \geq 2$, we can cast the eigenvalues into three classes $C_{1}^{n}, C_{2}^{n}, C_{3}^{n}$. They comprise, respectively, nondegenerated, twofold, and more than twofold degenerated eigenvalues.

A total of $D_{1}^{n}=2^{n-1}+1$ and $D_{2}^{n}=2^{n}$ eigenvalues are found in the classes $C_{1}^{n}$ and $C_{2}^{n}$. Eigenvalues in these classes do not appear again for any other value of $n$. However, for a given value of $n$, the eigenvalues remain in the proximity of the corresponding ones in the former generation of $n^{\prime}=n-1$. The total of remaining eigenvalues in the class is $D_{3}^{n}=\frac{3}{2}\left(3^{n-1}\right.$ $\left.-2^{n}+1\right)$. As the total number of eigenvalues increases with $3^{n}$, the relative number of states in $C_{1}^{n}$ and $C_{2}^{n}$ become vanishingly small as $n \rightarrow \infty$.

Contrary to what is observed with the two first classes, eigenvalues in $C_{3}^{n}$ are recurrent; i.e., once they are present in the spectrum for a given value of $n=q$, they will be found again for any $n>q$. Moreover, their degeneracy increases with $n$ in a very precise way. Therefore, the number of emerging eigenvalues at a given generation together with their degeneracy can be quantified according to the following scheme: the number of degenerate eigenvalues that emerge at generation $q$ is $2^{q-3}$; at generation $n$, the degeneracy of any eigenvalue that first appeared at generation $q$ is $d_{n, q}$ $=\frac{3^{n-q+2}-3}{2}$. Note that $d_{q, q}=3$ always. The first $C_{3}$ eigenvalues emerging at $q=3$ and 4 are, respectively, 0 and $\pm \sqrt{3}$. The large degeneracy indicates that the characteristic polynomial is highly factorized. Nevertheless, this relationship does not help much in finding exact roots of the polynomials when $q \geq 5$.

\section{RESULTS}

\section{A. Symmetry properties}

We have scrutinized all eigenstates until $n=9$, finding clear relationships among several of their properties and the classes to which the corresponding eigenvalue belongs. The most direct one refers to the way the states reflect the network invariance by $2 \pi / 3$ rotations around the central node. Let us define a paritylike property $P$ based on the value of $\sum_{i=1}^{N} a_{E, i}$, so that $P=$ odd (even) when this sum is zero (nonzero). We have found that, for any $P=$ even state, all sets of three equivalent sites, i.e., the three sites that are mapped onto one another by rotation of the network by $2 \pi / 3$ and $4 \pi / 3$ around the central site, share exactly the same amplitude $a_{E}$. The same amplitude is found for all sites that are mapped onto one another by inversion operations over the three bisectrices of the original triangle. All $C_{1}^{n}$ states are even and, conversely, all $C_{2}^{n}$ and $C_{3}^{n}$ states are odd.

However, we find two kinds of $P=$ odd states, depending on whether they belong to the $C_{2}^{n}$ or $C_{3}^{n}$ class. Irrespective of the particular class, the amplitude at the central site for any odd state vanishes identically, i.e., $a_{E, i=N(n-1)}=0$.

In Figs. 2(a)-2(c) we illustrate some features of three states when $n=2$. They correspond, respectively, to the largest eigenvalue $E_{7}$, which always belongs to $C_{n}^{(1)}$, and to the second largest twofold degenerated eigenvalue $E_{5}, E_{6}$ $=-(\sqrt{5}+1) / 2$, which is in the class $C_{2}^{(2)}$. As exemplary shown for $n=2$, the largest eigenvalue state has only positive components (absence of nodes). The two states corresponding to $E_{5,6}$ have odd parity and are clearly orthogonal.

The states in $C_{2}^{n}$ are such that, for each set of three equivalent sites (by rotation of the network by $2 \pi / 3$ and $4 \pi / 3$ around the central site), the sum of the amplitudes $a_{i}$ is zero. However, no inversion symmetry, as found for even states, is observed.

The states in $C_{3}^{n}$ do not have the same general rotation symmetry around the central site but a large number of local $2 \pi / 3$ rotational symmetries. Indeed, we found that, if the corresponding eigenvector appeared for the first time in $C_{3}^{q}$, at generation $n$ it contains $N(n-q+2)$ nodes with zero amplitudes, including the central one. For instance, when $n=4$, all 12 states corresponding to $E=0$, which appears for the first time when $n=q=3$, have $N(3)=16$ sites with vanishing amplitude. The three states corresponding to $E=\sqrt{3}$, which appears for $n=q=4$, have seven sites with vanishing amplitudes. The sites with vanishing amplitudes are exactly those sites that are present in the network at generation $n-q+2$. Further, each set of three sites that appears around these zero amplitude sites at generation $n-q+3$ has the property that the sum to the corresponding amplitudes is zero. This is illustrated in the Fig. 2(d), when $n=q=3$, for a state with eigenvalue $E=0$.

\section{B. Localization properties}

The localization properties of the quantum states were evaluated with the help of Eq. (3). The general features of 


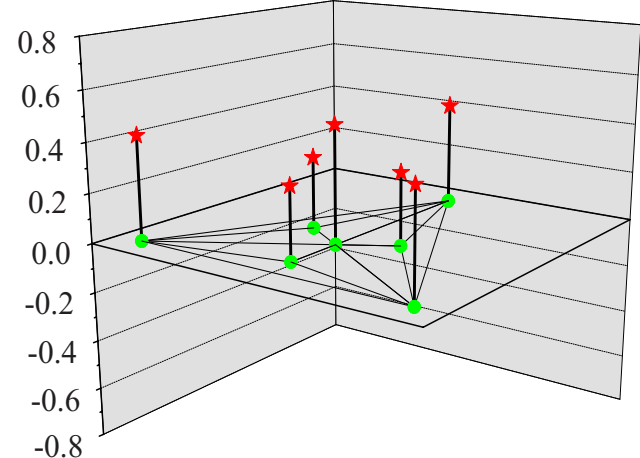

(a)

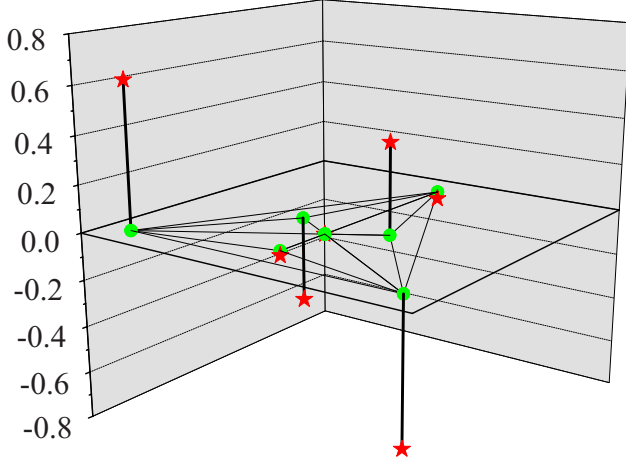

(b)

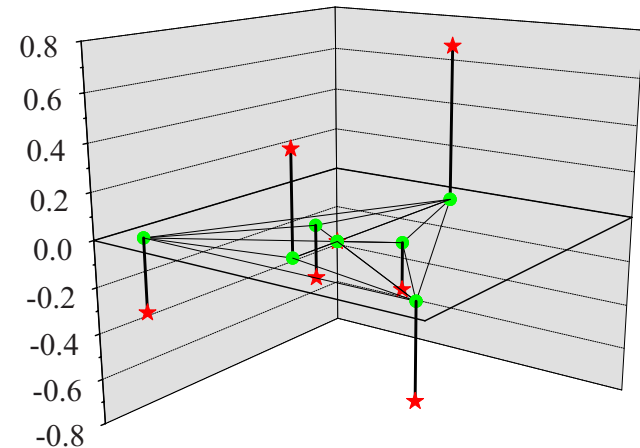

(c)

FIG. 2. (Color online) Illustration of parity properties for three eigenstates at generation $n=2$. Green (light gray) circles indicate AN nodes, while red (dark gray) stars indicate corresponding wave-function amplitude. (a) Even state corresponding to the largest value $E$ in class $C_{1}^{2}$. (b) and (c) Odd states corresponding to a twofold degenerate eigenvalue in class $C_{2}^{2}$.

the results are displayed in Fig. 3 where, in order to better compare the values of $\xi$ for different values of $n$, we draw the value of $\xi(i) / N(n)$ for individual states $i$. As the spectrum is highly degenerated, the label $i$ is chosen to be, in the first place, an increasing function of the energy. Then, within a degenerated level, the states are labeled according to increasing values of $\xi$. Since the horizontal axis has also been scaled to 1 by dividing $i / N$, the graphs show a recurrent form, with the presence of ever fine details as the value of $n$ increases.

So, despite the shown patterns seem quite irregular, it is possible to identify large windows where the value of $\xi / N$ increases monotonically. These structures correspond to the

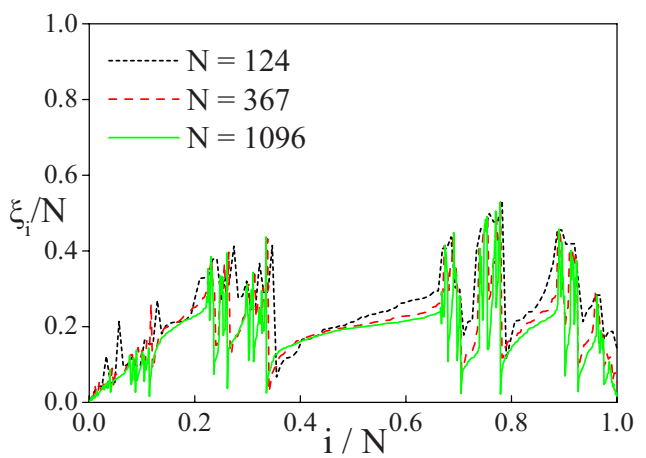

FIG. 3. (Color online) Dependence of $\xi_{i} / N$ with respect to $i / N$ for $n=5$ (black dots), 6 [red (dark gray) dashes], and 7 [green (light gray) solid line]. The states are labeled by increasing values of $E$ and, within each degenerate level, by increasing values of $\xi$. highly degenerate levels of $C_{3}^{n}$ class, where the individual states have been classified according to increasing values of $\xi$. This behavior makes it clear that the states corresponding to degenerate levels have own particular features.

The behavior of the states corresponding to the classes $C_{1}^{n}$ and $C_{2}^{n}$ is much difficult to be perceived in Fig. 3, as such states are immersed into the overwhelmingly larger number of $C_{3}^{n}$ states. However, the behavior of some of them, at specific positions in the spectrum, can be identified. For instance, the states corresponding to smallest and largest value of $E$, both in $C_{1}^{n} \forall n$, have very low values of $\xi$. On the other hand, $C_{1}^{n}$ and $C_{2}^{n}$ states that precede the large $C_{3}^{n}$ windows, like those just before $E=0$ and $\sqrt{3}$, are characterized by quite large values of $\xi$.

In Figs. 4(a) and 4(b), we plot the absolute value of local amplitudes for two $n=7$ states: $i=125$ and 244 , both with energy $E=0$ and, respectively, smallest and largest value of $\xi$. It is possible to visualize that the state with smallest value of $\xi$ is characterized by a few large amplitude spikes, distinguishing themselves from the much lower amplitudes in the remaining sites. This picture contrasts with that for $i=243$, where the amplitudes are more homogeneously distributed over all sites, evidencing properties of an extended state. Figure 4(c), for the $C_{2}^{n}$ state $i=286$, with energy $E \simeq 1.0495$, shows a pattern similar to that in Fig. 4(b), in agreement with the indication of an extended state $(\xi / N=0.4181)$.

To characterize the state properties in the $n \rightarrow \infty$ limit, it is necessary to follow the behavior of $\xi$ with $n$. As discussed before, truly extended states obey a linear dependence be- 

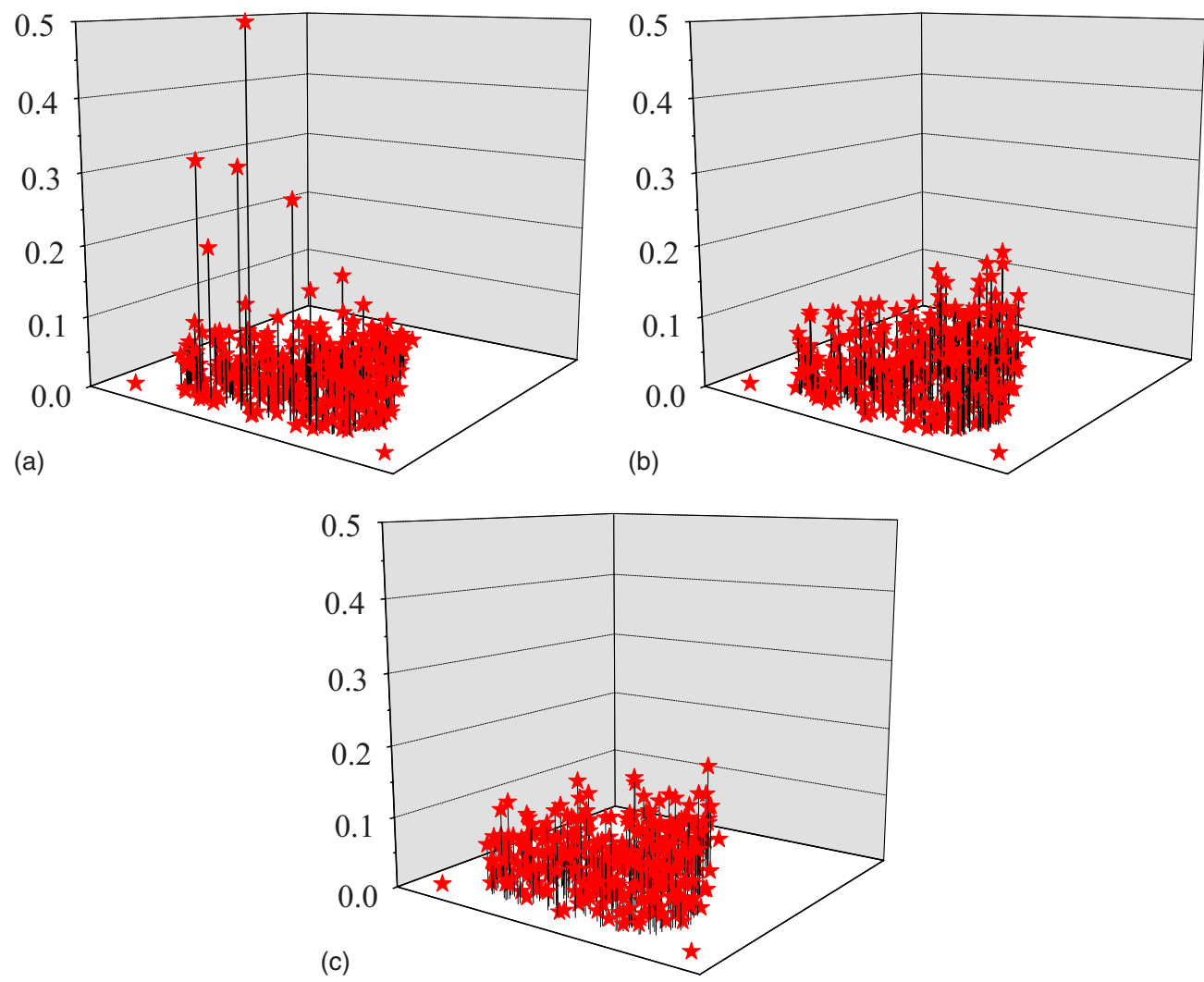

FIG. 4. (Color online) Absolute value of wave amplitudes for three states when $n=7$ indicated by red (dark gray) stars. (a) and (b) correspond to states with $E=0$ and, respectively, to smallest and largest values of $\xi$. In (c) we show the state with largest value of $\xi$ in the class $C_{2}^{7}$ and $E=\sqrt{3}$. The increasing value of $\xi$ stays in close correspondence with the trend to change from localized to extended character.

tween $\xi(n)$ and $N(n)$. However, as the number of states increases with the size of the system, a criterion must be defined to make the correspondence among the states in different generations $n$. For states in $C_{3}^{n}$, such correspondence is easier to be made, as the eigenvalues in one generation will be present in all further generations. As this does not happen for eigenvalues in $C_{1}^{n}$ and $C_{2}^{n}$, we will compare them by their relative positions to the eigenvalues in the class $C_{3}^{n}$.

In Fig. 5, we show the dependence of $\xi$ on the number of sites $N$ for some selected states, from which it is possible to evaluate the exponent $\alpha$ in $\xi \sim N^{\alpha}$. In Fig. 5(a), the two states with $E=0$ and smallest and largest values of $\xi$ are characterized by $\alpha=0.40$ and 0.92 , respectively. The indication of localized and extended character confirms the overall picture shown in Fig. 4. The result in Fig. 5(b), for the corresponding states of $E=-\sqrt{3}$, reveals a much smaller variation in the value of $\alpha \in[0.805,0.897]$ for the states corresponding to extreme values of $\xi$. Similar results are obtained for other values of $E$ in $C_{3}^{n}$, with a clear indication that, despite the occurrence of some localized states, the results in this class have a grater tendency of containing states with extended character.

States of the $C_{1}^{n}$ class can display both localized and extended characters as indicated by the sequence of values of $\xi$ for the largest and smallest eigenvalues and for the state with largest value of $\xi$. While small slopes $\alpha=0.051$ obtained for the sequence of smallest eigenvalues [Fig. 6(c)] indicate localization, the state with $E \simeq 1.239$, characterized by $\alpha \simeq 1$, which maximizes the value of $\xi$ in all generations, shows the opposite extended pattern. Similarly, states in class $C_{2}^{n}$ can display both localized and extended characters, as shown in Fig. 5(d). In the first one, the behavior of $\xi$ indicates localized nature for the pair of states corresponding to the second smallest eigenvalue, while extended properties are obtained for the pairs of states with $E \simeq 0.72$. In both cases, small values of $\alpha$ hint at a strong localization character. On the other hand, extended properties are found for the series of eigenvalues that precede the eigenvalue $E=\sqrt{3}$.

Since the relative number of states in classes $C_{1}^{n}$ and $C_{2}^{n}$ decreases with $n$, let us concentrate on the properties of $C_{3}^{n}$ states. A further characterization of their localization character can be provided by taking the average values of $\xi$ for the states with the same eigenvalue. For this purpose, for each value of $n$, the states to each degenerated eigenvalue have been divided into ten groups according to increasing values of $\xi$. Then, as in Fig. 5, we draw in Fig. 6(a) $\langle\xi(n)\rangle$ as a function of $N(n)$. The values of $\alpha$ for each subset show, consistently, a tendency to accumulate at $\alpha$ in the order of 1.0. Another way to confirm the result is to draw the participation ratio probability distribution $P(\xi / N)$ as a function of $\xi / N$ [see Fig. 6(b)]. For three increasing generations, the position of the peaks of $P$ indicates that the values of $\xi$ for $E=0$ states increase linearly with the size of the system, so that their extended nature is preserved in the limit of infinite system. These features support our claims of an overwhelming extended character of states in the uniform model. 

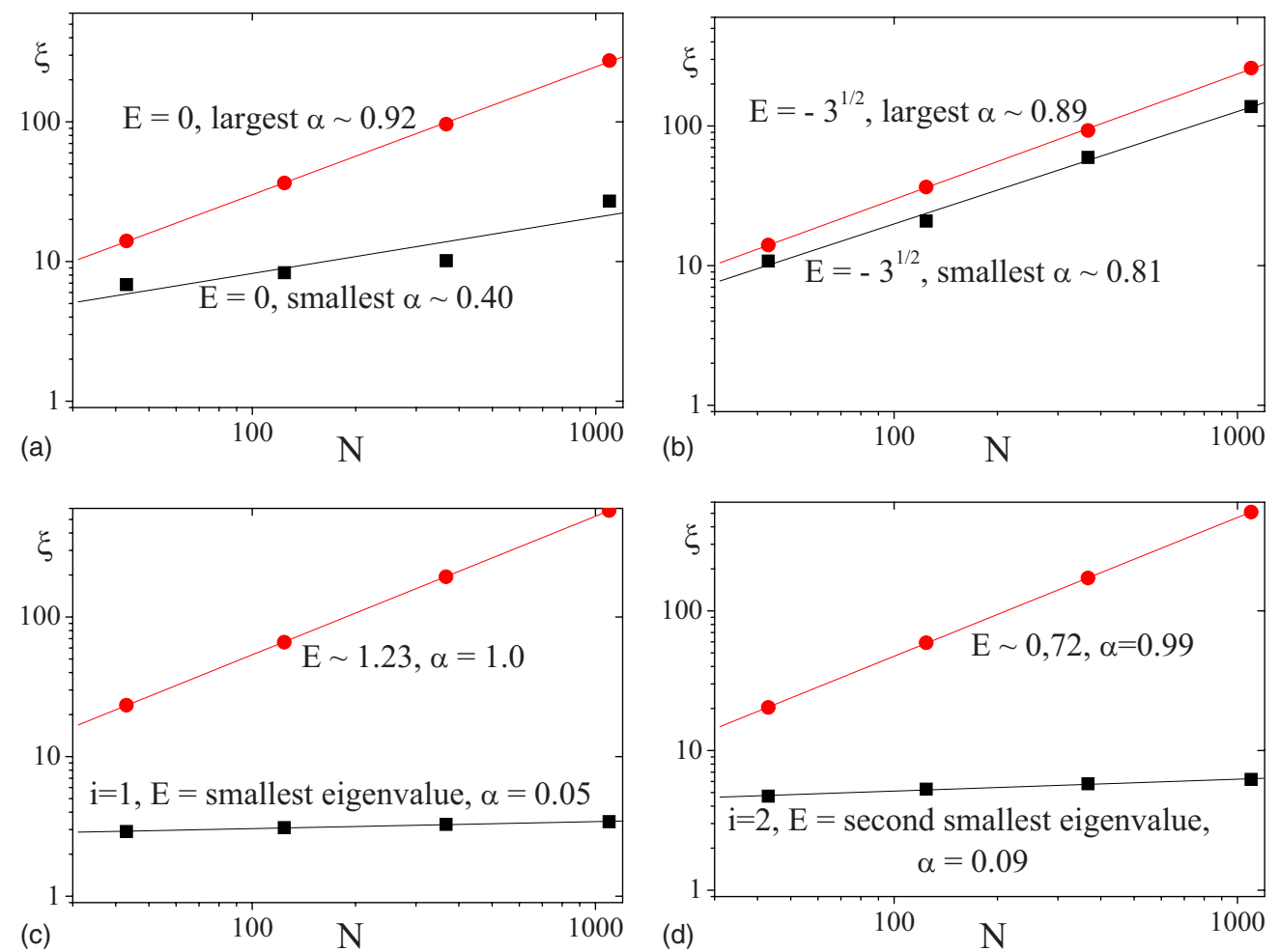

FIG. 5. (Color online) Participation ratio $\xi$ for some specific states, as a function of $N$, for generations $n=4,5$, 6, and 7. Red (dark gray) circles and black squares correspond, respectively, to states with large and low values of $\xi$. (a) $E=0$ and (b) $E=-\sqrt{3}$ correspond to states of class $C_{3}^{n}$. (c) and (d) illustrate localized and extended states belonging classes $C_{1}^{n}$ and $C_{2}^{n}$, respectively.

\section{Localization properties of the nonuniform model}

Let us now discuss the properties of nonuniform model mentioned in Sec. II, where $V(i, j)=1 / z^{\beta}$. Positive values of $\beta$ decrease the relative hopping probability from electrons in the largely connected hubs. So, in principle, we should expect a decrease in the electron mobility and a tendency for localized states. The opposite happens for negative values of $\beta$, where the hopping of electrons from the hubs is enhanced, strengthening the mobility. Since the $V(i, j)$ nonuniformity destroys the exact scheme based on three well-defined classes, it is now better to provide an analysis of the average behavior of the states rather than following them individually.

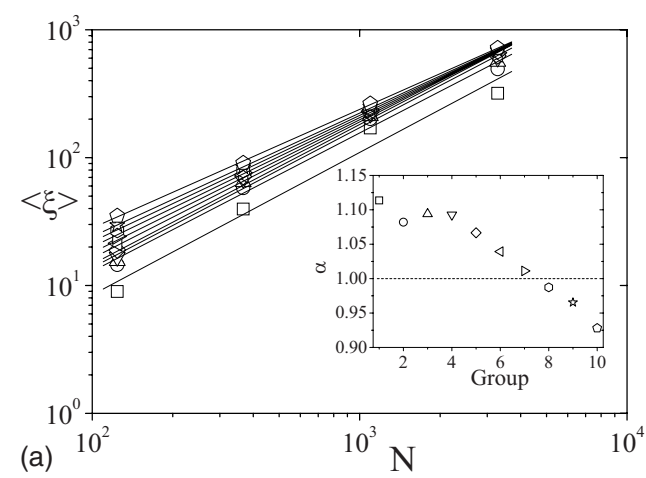

Our results are summarized in Fig. 7. For two distinct values of $\beta= \pm 1$, we qualitatively see, in Figs. 7(a) and 7(b), that the dependence of participation ratio $\xi / N$ with respect to $i / N$ has been distorted with respect to that of $\beta=0$ (Fig. 3). Nevertheless, it is still possible to identify the position of the $\beta=0$ highly degenerated $C_{3}^{n}$ eigenvalues. In order to quantify the change in the localization properties as function of $\beta$, we draw in Fig. 7(c) the behavior of the average value of the state slopes $\langle\alpha\rangle$ with respect to $\beta$. The abrupt change in the average value of $\langle\alpha\rangle$ at $\beta=3$ hints to an extended-localized transition of the wave function at this value. This behavior is in accordance with the discussion in the beginning of this subsection.

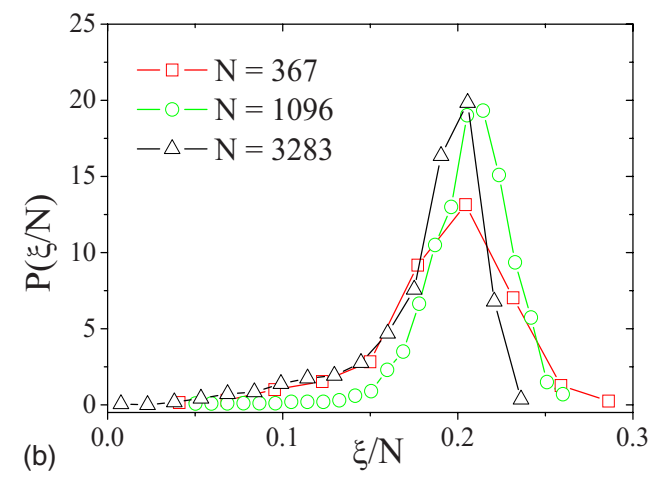

FIG. 6. (Color online) (a) Dependence on $N$ of the average participation ratio $\langle\xi\rangle$ for generations $n=5,6$, 7, and 8 . All states with energy $E=0$ have been cast into ten groups according to their values of $\xi$. The values of the different slopes, drawn in the inset, indicate that most states are extended. (b) The participation ratio probability distribution $P(\xi / N)$ of states with $E=0$, for $n=6$ [red (dark gray) squares], 7 [green (light gray) circles], and 8 (black triangles), is largely concentrated on large values of $\xi / N$ for all $n$. This is in accordance with (a). 

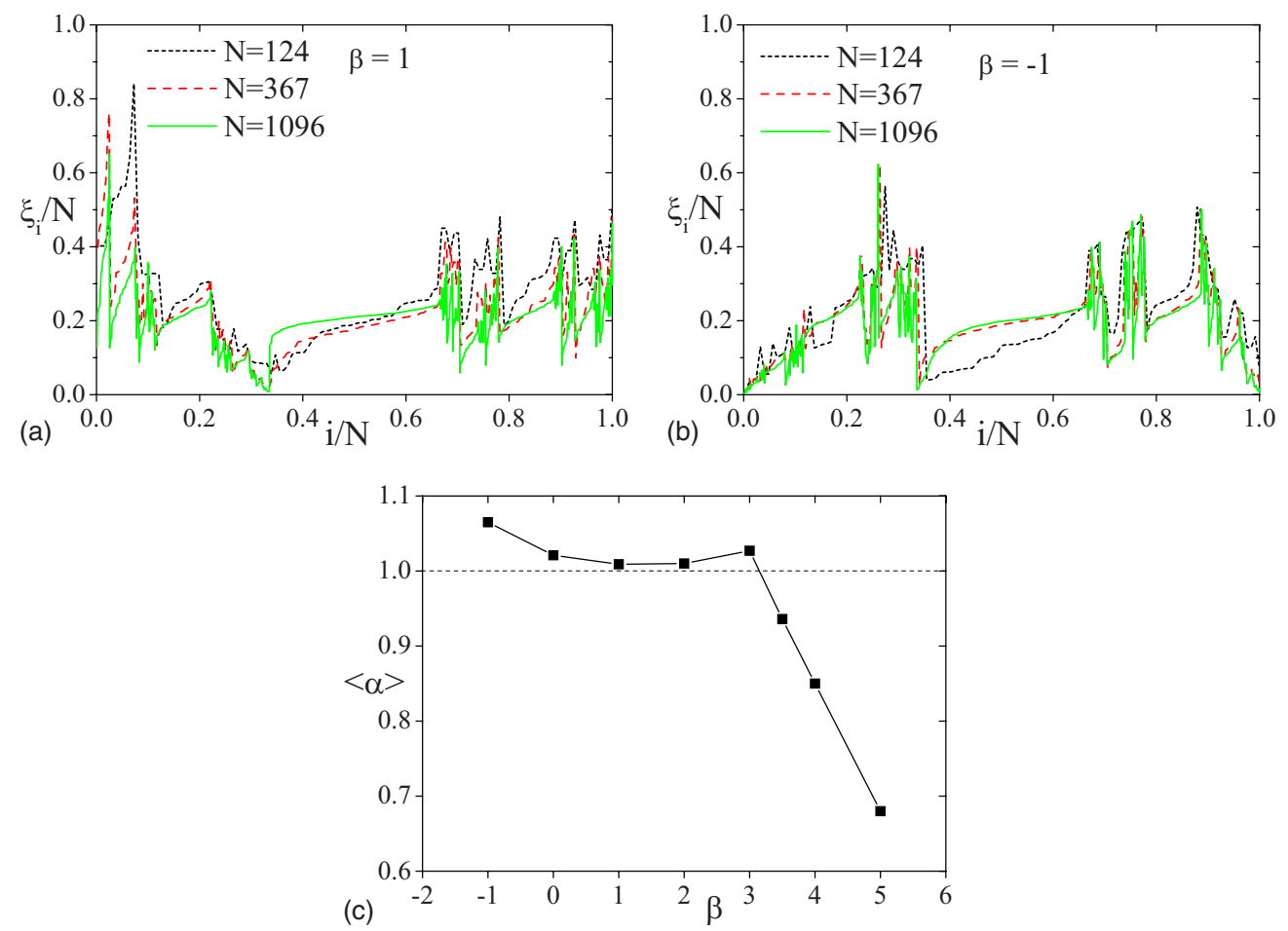

FIG. 7. (Color online) Dependence of $\xi_{i} / N$ with respect to $i / N$ for $n=5$ (black dots), 6 [red (dark gray) dashes], and 7 [green (light gray) solid line] when (a) $\beta=1$ and (b) $\beta=-1$. The states are labeled by increasing values of $E$. In (c), we show the dependence of $\langle\alpha\rangle$ with respect to $\beta$, with a clear indication of a sudden change in the nature of the states for $\beta=3$.

\section{Anderson transition}

In this section we briefly point out the most important features of the eigenstates that result from introducing disorder into the tight-binding model. In this case, the on-site energy $\epsilon_{i}$ is considered as a random independent variable, describing the local disorder that disturbs the motion of electrons. $\epsilon_{i}$ is considered as a variable characterized by a probability function $P\left(\epsilon_{i}\right)=\Phi\left(\Delta / 2-\left|\epsilon_{i}\right|\right) / \Delta$, with $\Phi$ being the step function. The parameter $\Delta$ is a measure for the disorder strength and the main purpose is to identify the possible existence of a Anderson transition for some finite value of $\Delta$.

As observed in the discussion of the nonuniform model, the introduction of the disorder breaks the high degree of degeneracy of the $C_{3}^{n}$ states, as well as the three classes scheme observed in the energy spectrum of the uniform model. Thus, we resort again to a discussion of the average properties of the states as a dependency of the energy interval.

The Anderson transition has been studied taking the average values of $\xi$ of the states in a given energy interval $|E|$ $\leq \delta E$, with $\delta E=0.001$. The results are summarized in Table I, where we present the obtained values of $\langle\alpha\rangle$ as function of $\Delta$. The results have been obtained by performing $M$ different realizations of disorder where, respectively, $M=1000,300$, and 50 for $n=6,7$, and 8 .

The results in Table I can be compared with those obtained for similar models built on complex network structures. Indeed, Anderson transition investigations on scalefree networks have shown that the localization of the electronic states is influenced by the connectivity of the net- work. Thus, the fractal dimension quantifies the critical disorder. ${ }^{20,21}$ Our investigation confirms the possibility of the absence of an Anderson transition at highly connected networks, as observed in Ref. 20.

As discussed in Sec. II, AN is quite distinct from other well-studied networks. ${ }^{16}$ Therefore, the current results can only be compared to those for other connected networks with some care. A natural way to generate surrogates that are relatively close to AN consists in removing or adding bonds to AN itself. In fact, we also explored this procedure but noticed that it interferes in a much more drastic way in the nature of the present states. The addition of this kind of disorder makes the results much more difficult to be analyzed then by adding on-site energies..

\section{CONCLUSIONS}

In this work we presented a very broad investigation of the properties of the eigenstates of a tight-binding model on

TABLE I. Dependence of the localization parameter $\alpha$ on disorder strength $\Delta$. For all investigated values of $\Delta$, a strong reduction in the value of $\alpha$ in comparison to the uniform model is noticed, indicating complete state localization.

\begin{tabular}{cc}
\hline \hline$\Delta$ & $\alpha$ \\
\hline 0.01 & 0.61 \\
0.1 & 0.42 \\
0.2 & 0.38 \\
0.5 & 0.36 \\
\hline
\end{tabular}


the Apollonian network. We considered the uniform model and two further model versions, where we could investigate, respectively, the influence of nonuniformity induced by the network geometry and by random on-site energies in the eigenstate properties.

In the regular case, we could establish a precise relationship between the three different classes in which the eigenvalue spectrum can be divided and parity properties of the corresponding eigenvectors. We have shown that localization property is not related to the eigenvalue classes, by the identification of eigenstates, with localized and extended properties, within each of the classes. However, as the number of states in the class $C_{3}^{n}$ increases much faster with $n$ than those of the other two classes, we proceeded with a quantification of the average participation ratio of the $C_{3}^{n}$ states. The results indicate a scenario in which extended states dominate the global behavior of the system.

By including an explicit dependence of the node degree on their ability to decrease or increase the electron mobility, we have shown that the general state properties can change from the quoted extended character into a localized one. Our results were based on the ansatz that the interaction strength decays with the node degree according to a power law. It was possible to obtain a positive critical value for the change in the property of the states.
The model has shown to be much more sensitive to state localization when random on-site energies are assigned to each site. Our results suggest that, for any nonzero amount of randomness, all states, even those in the neighborhood of the band center, assume localized nature.

As a final comment, we note that intermediate values of $\alpha$, i.e., far from the limiting values of 0 and 1 that characterize the exponentially localized and uniformly extended states are also abundant for all of considered models. Such "critical" states, as referred by other authors, ${ }^{22}$ usually display fractal-like properties that distinguish them from the states in the just described limiting cases.

Physical models constructed on ANs are known to produce quite unusual properties. These are due mostly on the existence of large degree nodes as well as with the existence of large number of loops. Because of this, it is not possible to affirm that other nonuniform disordered models on AN will not present other unexpected features.

\section{ACKNOWLEDGMENTS}

This work was partially supported by CNPq. The authors acknowledge fruitful discussions with H. J. Herrmann, J. S. Andrade Jr., and E. L. Albuquerque. RFSA thanks H. J. Herrmann for his hospitality at ETH.
${ }^{1}$ D. Belitz and T. R. Kirkpatrik, Rev. Mod. Phys. 66, 261 (1994).

${ }^{2}$ M. E. J. Newman, A.-L. Barabási, and D. J. Watts, The Structure and Dynamics of Networks (Princeton University Press, Princeton, NJ, 2006).

${ }^{3}$ S. Boccaletti, V. Latora, Y. Moreno, M. Chavez, and D.-U. Hwang, Phys. Rep. 424, 175 (2006)

${ }^{4}$ L. F. Costa, F. A. Rodrigues, G. Travieso, and P. R. Villas Boas, Adv. Phys. 56, 167 (2007).

${ }^{5}$ C. V. Giuraniuc, J. P. L. Hatchett, J. O. Indekeu, M. Leone, I. Perez Castillo, B. Van Schaeybroeck, and C. Vanderzande, Phys. Rev. E 74, 036108 (2006).

${ }^{6}$ J. S. Andrade, Jr., H. J. Herrmann, R. F. S. Andrade, and L. R. da Silva, Phys. Rev. Lett. 94, 018702 (2005).

${ }^{7}$ R. F. S. Andrade and H. J. Herrmann, Phys. Rev. E 71, 056131 (2005).

${ }^{8}$ A. M. C. Souza and H. J. Herrmann, Phys. Rev. B 75, 054412 (2007).

${ }^{9}$ A. P. Vieira, J. S. Andrade, Jr., H. J. Herrmann, and R. F. S. Andrade, Phys. Rev. E 76, 026111 (2007).

${ }^{10}$ G. R. Newkome, P. Wang, C. N. Moorefield, T. J. Cho, P. Mo- hapatra, S. Li, S. H. Hwang, O. Lukoyanova, L. Echegoyen, J. A. Palagallo, V. Iancu, and S. W. Hla, Science 312, 1782 (2006).

${ }^{11}$ R. F. S. Andrade and J. G. V. Miranda, Physica A 356, 1 (2005).

${ }^{12}$ F. Harary, Graph Theory (Perseus Books, Cambridge, 1995).

${ }^{13}$ S. N. Dorogovtsev, A. V. Goltsev, J. F. F. Mendes, and A. N. Samukhin, Phys. Rev. E 68, 046109 (2003).

${ }^{14}$ J. P. K. Doye and C. P. Massen, Phys. Rev. E 71, 016128 (2005).

${ }^{15}$ H. J. Herrmann, G. Mantica, and D. Bessis, Phys. Rev. Lett. 65, 3223 (1990).

${ }^{16}$ L. F. Costa and R. F. S. Andrade, New J. Phys. 9, 311 (2007).

${ }^{17}$ D. J. Watts and S. H. Strogatz, Nature (London) 393, 440 (1998).

${ }^{18}$ A. L. Barabasi and R. Albert, Science 286, 509 (1999).

${ }^{19}$ P. W. Anderson, Rev. Mod. Phys. 50, 191 (1978).

${ }^{20}$ M. Sade, T. Kalisky, S. Havlin, and R. Berkovits, Phys. Rev. E 72, 066123 (2005).

${ }^{21}$ M. Schreiber and H. Grussbach, Phys. Rev. Lett. 76, 1687 (1996).

${ }^{22}$ H. Q. Yuan, U. Grimm, P. Repetowicz, and M. Schreiber, Phys. Rev. B 62, 15569 (2000). 